\title{
Tubeimoside I induces accumulation of impaired autophagolysosome against cervical cancer cells by both initiating autophagy and inhibiting lysosomal function
}

\author{
Xuping Feng ${ }^{1,2}$, Jing Zhou ${ }^{2}$, Jingyi $\mathrm{Li}^{3}$, Xueyan Hou${ }^{4}$, Longhao Li ${ }^{1,5}$, Yongmin Chen ${ }^{6}$, Shuyue Fu' ${ }^{2}$ Li Zhou ${ }^{2}$, \\ Changlong $\mathrm{Li}^{7}$ and Yunlong Lei ${ }^{1}$
}

\begin{abstract}
Cervical cancer is one of the most aggressive human cancers with poor prognosis due to constant chemoresistance and repeated relapse. Tubeimoside I (TBM) has been identified as a potent antitumor agent that inhibits cancer cell proliferation by triggering apoptosis and inducing cell cycle arrest. Nevertheless, the detailed mechanism remains unclear and needs to be further elucidated, especially in cervical cancer. In this study, we found that TBM could induce proliferation inhibition and cell death in cervical cancer cells both in vitro and in vivo. Further results demonstrated that treatment with TBM could induce autophagosome accumulation, which was important to TBM against cervical cancer cells. Mechanism studies showed that TBM increased autophagosome by two pathways: First, TBM could initiate autophagy by activating AMPK that would lead to stabilization of the Beclin1-Vps34 complex via dissociating Bcl-2 from Beclin1; Second, TBM could impair lysosomal cathepsin activity and block autophagic flux, leading to accumulation of impaired autophagolysosomes. In line with this, inhibition of autophagy initiation attenuated TBMinduced cell death, whereas autophagic flux inhibition could exacerbated the cytotoxic activity of TBM in cervical cancer cells. Strikingly, as a novel lethal impaired autophagolysosome inducer, TBM might enhance the therapeutic effects of chemotherapeutic drugs towards cervical cancer, such as cisplatin and paclitaxel. Together, our study provides new insights into the molecular mechanisms of TBM in the antitumor therapy, and establishes potential applications of TBM for cervical cancer treatment in clinic.
\end{abstract}

Correspondence: Yunlong Lei (leiyunlong@126.com)

'Department of Biochemistry and Molecular Biology, and Molecular Medicine and Cancer Research Center, Chongqing Medical University, Chongqing 400016, P.R. China

${ }^{2}$ State Key Laboratory of Biotherapy and Cancer Center, West China Hospital, and West China School of Basic Medical Sciences \& Forensic Medicine, Sichuan University, and Collaborative Innovation Center for Biotherapy, Chengdu 610041, P.R. China

Full list of author information is available at the end of the article. These authors contributed equally: Xuping Feng, Jing Zhou, Jingyi Li Edited by B. Zhivotovsky

\section{Introduction}

With 500,000 incident cases and 260,000 deaths annually, cervical cancer has been implicated one of the most common cancers worldwide ${ }^{1,2}$. Primary preventions and early treatment of precancerous lesions have sharply declined the incidence rate in most developed countries; however, the morbidity and mortality remain high in some low-income countries ${ }^{3,4}$. In addition, the primary methods for cervical cancer treatment such as surgery, radiotherapy and adjuvant chemotherapy, have greatly

\section{() The Author(s) 2018}

(c) (i) Open Access This article is licensed under a Creative Commons Attribution 4.0 International License, which permits use, sharing, adaptation, distribution and reproduction cc) in any medium or format, as long as you give appropriate credit to the original author(s) and the source, provide a link to the Creative Commons license, and indicate if changes were made. The images or other third party material in this article are included in the article's Creative Commons license, unless indicated otherwise in a credit line to the material. If material is not included in the article's Creative Commons license and your intended use is not permitted by statutory regulation or exceeds the permitted use, you will need to obtain permission directly from the copyright holder. To view a copy of this license, visit http://creativecommons.org/licenses/by/4.0/. 
improved the carcinoma survival rate ${ }^{5,6}$. Nonetheless, increasing radioresistance or chemoresistance, repeated relapse and tumor metastasis limit the treatment efficacy, highlighting the urgency of developing novel and reliable therapeutic strategies.

Autophagy is a conservative lysosomal degradation pathway during which the intracellular materials are degraded and recycled ${ }^{7}$. Cellular stress events, such as energy limiting, oxidative stress and nutrient deprivation, result in accumulation of damaged or toxic proteins and organelles that can drive autophagy to sustain cellular homeostasis ${ }^{8}$. The autophagic products, such as amino acids, fatty acids and other small molecules can provide a certain amount of energy and synthetic substrates to maintain adequate energy. Given its "selfdigest" function, the role of autophagy in cancer is complex and context-dependent ${ }^{9}$. Autophagy is originally known as a tumor suppressor from the investigation of the tumorigenesis tendency in mice with allelic loss of autophagy-related genes (ATGs). However, increasing studies have implicated its role in tumor promoting by assisting cancer cells survival in stress either from environment or induced by tumor therapy ${ }^{10,11}$. Targeting the autophagy process has been regarded as a novel therapeutic approach ${ }^{12}$. Therefore, development of novel autophagy modulator has rewired a way of cancer treatment.

Tubeimoside I (TBM) is extracted from the tuber of Bolbostemma paniculatum (Maxim) Franquet (Cucurbitaceae), a traditional Chinese herb previously used in anti-viral or anti-inflammatory treatment ${ }^{13}$. Recently, growing studies have reported its direct cytotoxity in multiple human cancer cells, characterized by mitochondrial damage, endoplasmic reticulum stress, apoptosis and cell cycle arrest ${ }^{14-17}$. In addition, TBM could sensitize human ovarian cancer cells to cisplatin $(\mathrm{CDDP})^{18}$. TBM has been considered as a promising anticancer agent. However, the underlying mechanism remains unclear and elusive.

In the present study, we found that TBM-treated cervical cancer cells displayed decreased proliferating rate and obvious cell death. TBM also promoted remarkable autophagosome synthesis, resulted from activation of adenosine monophosphate-activated protein kinase (AMPK) signaling. In addition, autophagic flux was blocked in the late stage of autophagic process, eventually leading to impaired autophagolysosomes accumulation and cell death. Moreover, this novel autophagic cell death inducer may enhance the treatment efficacy of chemotherapeutic drugs towards cervical cancer. Our findings suggest that TBM act as a potent autophagy modulator and may provide new insights into therapeutic strategy for cervical cancer.

\section{Results}

TBM inhibits cervical cancer cells proliferation both in vitro and in vivo

To identify the role of TBM in cervical cancer, cervical cancer cell lines (HPV18-positive HeLa and HPV16positive $\mathrm{SiHa}$ ) were treated with TBM. MTT assay showed that TBM markedly decreased the cervical cancer cells' viability in a dose-dependent manner (Fig. 1a). LDH release assay also revealed that TBM could damage the integrity of plasma membrane (Fig. 1b). As shown in Supplementary Figure 1, cells exposed to TBM exhibited a significant survival inhibition, as evidenced by the decreased colony numbers. Furthermore, in comparison to controls, a notably lower rate of EdU-postive cells was observed in TBM-treated cells (Figs. 1c, d), indicating the growth inhibitory effect of TBM on cervical cancer cells.

To further define the antitumor effects of TBM in vivo, we established a mouse xenograft model with HeLa cells, following by receiving TBM or saline solution treatment. Tumor size, volume and mass increased dramatically in the vehicle control. In contrast, tumor in TBM-treated mice grew less prominent (Figs. 1e-g). In addition, most of the TBM-treated tumors displayed reduced Ki67 staining (Figs. 1h, i), indicating the decreased proliferating ability in TBM-treated group. Collectively, these results suggest that TBM inhibit proliferation of cervical cancer cells both in vitro and in vivo.

Besides, we found that TBM effectively decreased the cell viability of several cancer cells in a dose-dependent manner, including glioblastoma, breast cancer, hepatocarcinoma, lung cancer and colorectal cancer cells, indicating that TBM is a broad-spectrum antineoplastic agent (Supplementary Figure 2). Unfortunately, TBM could also induce growth inhibition in normal cells (Supplementary Figure $3 \mathrm{~A}$ and $3 \mathrm{~B}$ ). However, the cytotoxicity of TBM was low when treatment concentration was less than $10 \mu \mathrm{M}$, and the cytotoxicity of TBM was equivalent to commonly used anticancer drugs such as cisplatin (CDDP), paclitaxel (PTX), doxorubicin (DOX) and 5-fluorouracil (5-FU) (Supplementary Figure 3C), suggesting that low concentration of TBM is relatively safe and may be benefit for cancer treatment.

\section{TBM induces apoptosis in cervical cancer cells both in vitro and in vivo}

To get more insights into the mode of TBM-induced cell death, we treated cervical cancer cells with TBM combination with a series of death inhibitors. As shown in Figs. 2a, b, Z-VAD-FMK, a pan-caspase inhibitor ${ }^{19}$, partially rescued TBM-induced cell death; in contrast, other inhibitors including ferrostatin-1 and necrostatin-1, failed to influence the cell death caused by TBM, in spite of their specific capacities to inhibit ferroptosis ${ }^{20}$ and necropto$\mathrm{sis}^{21}$, respectively. This implies that apoptosis might be 

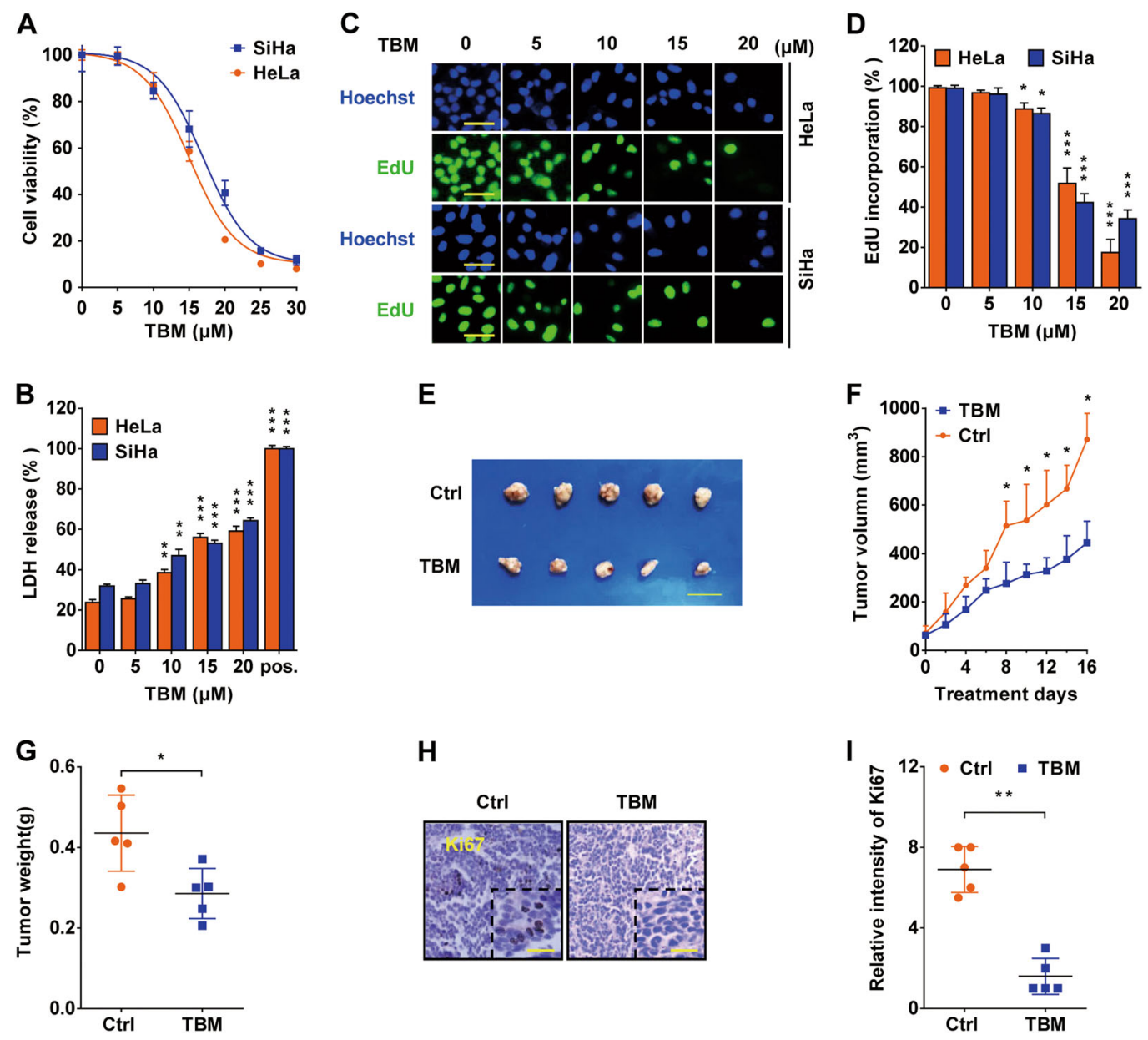

Fig. 1 TBM inhibits cervical cancer cells proliferation. a Hela and SiHa cells were treated with indicated concentrations of TBM for $24 \mathrm{~h}$. Cell viability was measured by the MTT assay. $\mathbf{b}$ TBM disrupted cellular membrane integrity as measured by LDH release in the medium. Cells were treated as in (a). c-d Cell proliferation of HeLa and SiHa cells were measured by EdU labeling. Cells were treated as in (a). Scale bars: 100 $\mu$ m. e-g Nude mice bearing HeLa xenograft tumor were treated with $100 \mu \mathrm{L}$ saline solution (control, $n=5$ ) or $3 \mathrm{mg} / \mathrm{kg}$ TBM $(n=5)$ daily for 16 days. e Tumor tissues were taken and imaged after animals sacrificed. Scale bars: $1 \mathrm{~cm}$. f Tumor volumes were monitored every other day and calculated by the length and width. $\mathbf{g}$ Tumors were weighed immediately once mice were killed. $\mathbf{h}-\mathbf{i}$ Tumor tissues were sectioned and subjected to immunohistochemistry for evaluating expression of Ki67. Scale bars: $100 \mu \mathrm{m} .{ }^{*} p<0.05 ;{ }^{* *} p<0.01 ;{ }^{* *} p<0.001$

associated with TBM-induced cervical cancer cell death. Consistently, both TUNEL assay and flow cytometry analysis exerted prominent apoptotic effect in cervical cancer cells (Figs. 2c-e). In addition, cleaved-CASP3 and cleaved-PARP1, one of caspase downstream effectors ${ }^{22}$, were accumulated upon TBM treatment (Fig. 2f). Consistently, TBM also caused apoptosis in cervical cancer in vivo (Fig. 2g). In summary, these data indicate that apoptosis is involved in TBM against cervical cancer both in vitro and in vivo.

\section{TBM induces autophagosome formation in cervical cancer cells}

Interestingly, as shown in Figs. 2a, b, co-treatment of TBM and CQ could initiate more cell death than TBM alone, indicating that autophagy might be also involved in TBM-induced cell death. To explore the relationship between TBM and autophagy, cervical cancer cells were treated with TBM and then LC3 II conversion, a specific marker of autophagy ${ }^{23}$, was measured. Of note, TBM significantly induced LC3 II accumulation in both dosedependent and time-dependent manners (Figs. 3a, b). In addition, transmission electron microscopy experiment also showed that the formation of double-membraned autophagic vacuoles was frequently observed in cervical cancer cells treated with TBM (Supplementary Figure 4). Furthermore, both the endogenous LC3 and exogenous GFP-LC3 puncta, representing the number of autophagic vacuoles $^{23}$, were remarkably increased in TBM-treated cells (Figs. $3 \mathrm{c}-\mathrm{f}$ ). Acidic vesicular organelles, which are 


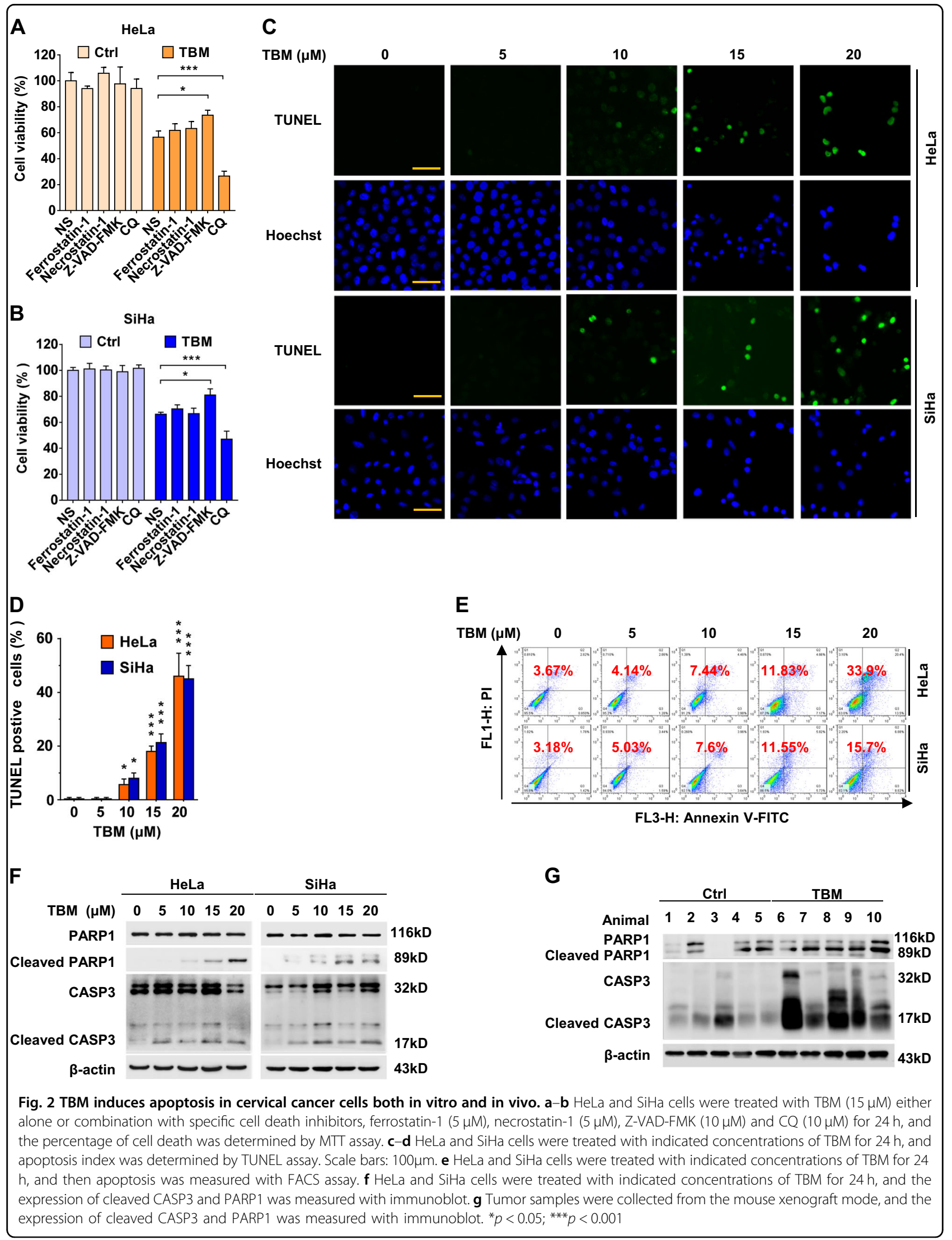




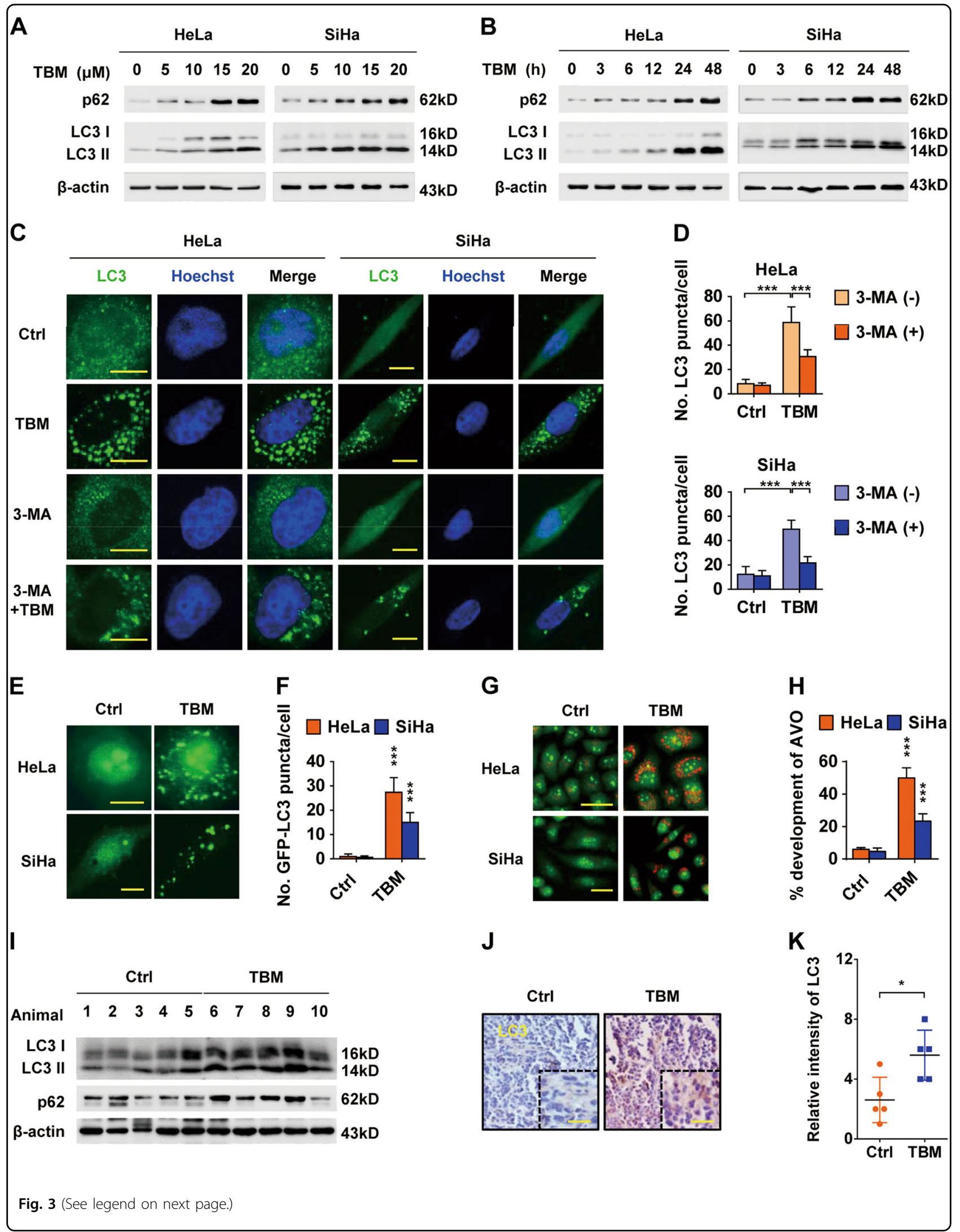


(see figure on previous page)

Fig. 3 TBM initiates autophagosome formation in cervical cancer cells. a Immunoblot analysis of LC3 and p62 in HeLa and SiHa cells treated with indicated concentrations of TBM for $24 \mathrm{~h}$. $\mathbf{b}$ Immunoblot analysis of LC3 and p62 in HeLa and SiHa cells treated with $15 \mu \mathrm{M}$ TBM for indicated time. c Immunofluorescence analysis of endogenous LC3 puncta formation in TBM-treated HeLa and SiHa cells in present with or without 3-MA for $24 \mathrm{~h}$. Scale bar: $20 \mu \mathrm{m}$. $\mathbf{d}$ Graph shows quantification of LC3-positive punctate cells in (c). e HeLa and SiHa cells were transfected with GFP-LC3 for 48

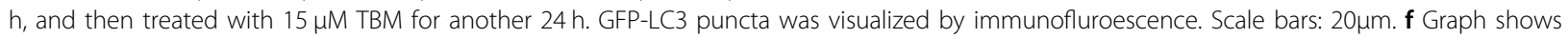
quantification of GFP-LC3-positive punctate cells in (e). $\mathbf{g}$ Acridine orange was used to stain acidic vesicular organelles in HeLa and SiHa cells treated with $15 \mu$ M TBM. Scale bars: $50 \mu \mathrm{m}$. $\mathbf{h}$ The total number of acidic vesicular organelles (AVO) per cell in Figure G was quantified by ImageJ software. $\mathbf{i}$ Tumor tissues were hydrolyzed, and then LC3 and p62 were examined by immunoblot. $\mathbf{j}-\mathbf{k}$ Tumor tissues were resectioned and subjected to immunohistochemistry for evaluating expression of LC3 in mouse tumor xenograft. Scale bars: $100 \mu \mathrm{m} .{ }^{*} p<0.05 ;{ }^{* * *} p<0.001$

markers of autophagosomes ${ }^{24}$, were also markedly increased following TBM treatment (Figs. 3g, h), highlighting its capacity in inducing autophagosome accumulation.

To examine whether TBM induces autophagy in vivo, tumor samples were analyzed for LC3 II level. We observed an increase of LC3 II conversion by immunoblot analysis (Fig. 3i). Consistently, TBM-treated xenografts displayed stronger LC3 staining compared with the control group (Figs. 3j, k). In summary, TBM can induce autophagosome accumulation in cervical cancer both in vitro and in vivo. In addition, TBM also induced autophagosome accumulation in various types of cancer cells as evidenced by the LC3 II accumulation (Supplementary Figure 5).

\section{TBM initiates autophagy by activating AMPK}

Once autophagy initiated, a series of ATGs will drastically be activated to mediate autophagosome formation. A key event in the autophagy pathway is the activation of the Beclin1-Vps34 complex, which functions in the formation of isolated membrane (also known as the phagophore). Intriguingly, we found that Beclin1 showed a relatively strong interaction with ATG14 and weak binding affinity toward Bcl-2 in TBM-treated cells (Figs. 4a-d), suggesting that TBM could stabilize the Beclin1-Vps34 complex by dissociating Bcl-2 from Beclin1. In addition, treated with TBM combination with 3-MA or wortmannin (WTM), the PtdIns3K inhibitors that selectively block activation of Beclin1-Vps34 complex ${ }^{25}$, obviously decreased LC3 II and LC3 dots formation in cervical cancer cells (Figs. 3c, d; Supplementary Figure 6A and 6B). Furthermore, molecular inhibition of ATG5 or Beclin1 by siRNA revealed a decrease in LC3 II accumulation (Supplementary Table 1, Figs. 4e, f). These results show that TBM can initiate autophagy in vitro.

AMPK and mTOR signaling pathways are two main autophagy-initiating pathways ${ }^{26}$. Considering that TBM could damage the mitochondria and cause the depletion of mitochondrial transmembrane potential $(\Delta \Psi \mathrm{m})^{27,28}$, which subsequently decreased the production of energy in the form of adenosine triphosphate (ATP), we hypothesized that AMPK might play a critical role in TBM-induced autophagy. To confirm this, we firstly measured the ATP levels in HeLa and SiHa cells treated with TBM. As expected, both cells exerted a dosedependent decrease of ATP production (Fig. 4g). Immunoblot also showed that TBM could activate AMPK and its down-stream protein, ACC, in a dose-dependent manner (Fig. 4h). To further exam the role of AMPK in TBM-induced autophagy, CC, a specific AMPK inhibitor, was used to inactivate $\mathrm{AMPK}^{29}$. Of note, CC decreased TBM-induced accumulation of LC3 II and formation of LC3 puncta (Figs. 4i, k). In summary, these data indicate that TBM initiates autophagy by activating AMPK in cervical cancer cells.

\section{TBM inhibits autophagic flux in cervical cancer cells}

In addition to initiate autophagy, our earlier observations also showed that TBM induced an increase of p62 (Figs. 3a, b, i), a substrate of autophagy which is delivered to the lysosomes for degradation ${ }^{8}$. Enhanced p62 expression can be either associated with increased protein synthesis or due to discontinuity of autophagosome turnover $^{30}$, suggesting that TBM may inhibit autophagic flux. To clarify the underlying mechanism, HeLa and $\mathrm{SiHa}$ cells were treated with TBM in combination with Baf A1 (a V-ATPase inhibitor) ${ }^{31}$ or CQ (a lysosomotropic compound $)^{32}$, both of which could block the end stage of autophagy. As shown in Figs. 5a-d, Baf A1 or CQ induced much less further accumulation of LC3 II protein and LC3 dot numbers in TBM-treated cells compared with control cells, supporting the inference that TBM may inhibit autophagic flux. Additionally, TBM treatment resulted in accumulation of p62 dots and increased colocalization with LC3 punta (Figs. 5e, f), which also suggested autophagic flux inhibition. Furthermore, autophagic flux induced by rapamycin could also be impeded by TBM, suggesting TBM-induced autophagic flux inhibition is independent on the mechanisms of TBM-initiated autophagy (Fig. 5g). Finally, tandem mCherry-GFP-LC3 reporter assays also determined TBM-induced autophagic flux inhibition evidenced by that exposure to TBM caused notable formation of yellow 
A

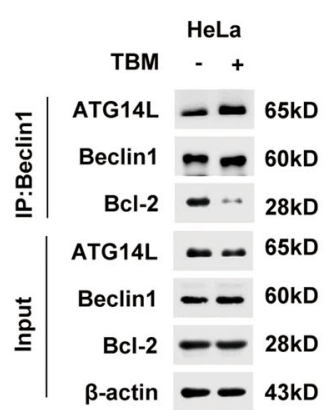

C

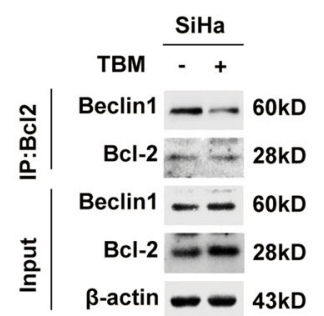

G

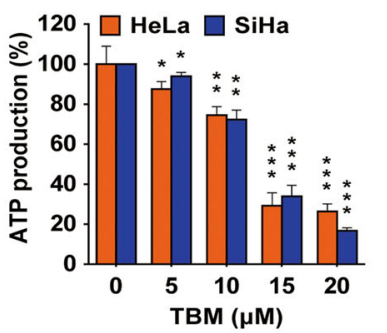

$\mathbf{J}$

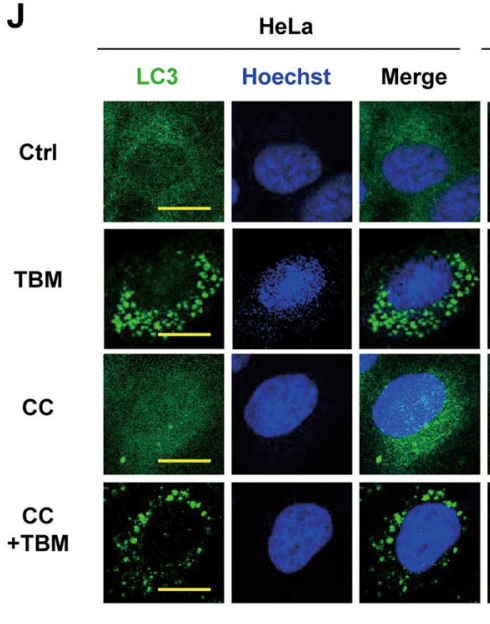

Fig. 4 TBM induces an AMPK-dependent autophagosome formation. a-b Interaction among Beclin1, Atg14L and BCl-2 in HeLa (a) and SiHa (b) cells was determined by co-immunoprecipitation assay. c-d Interaction between BCl-2 and Beclin1 in HeLa (c) and SiHa (d) cells was determined by co-immunoprecipitation assay. e HeLa and SiHa cells were transfected with siScramble or siATG5 for $48 \mathrm{~h}$, and then treated with $15 \mu \mathrm{M}$ TBM for another $24 \mathrm{~h}$. Expression of Atg5 and LC3 was examined by immunoblot. f HeLa and SiHa cells were transfected with siScramble or siBeclin 1 for $48 \mathrm{~h}$, and then treated with $15 \mu \mathrm{M}$ TBM for another $24 \mathrm{~h}$. Expression of Beclin1 and LC3 was examined by immunoblot. $\mathbf{g}$ ATP production was detected in cells treated with indicated concentrations of TBM by ATP Assay Kit (Beyotime Biotechnology, S0026). (h) Immunoblot analysis AMPK, ACC, AMPK phosphorylation (Thr172) and ACC phosphorylation (Ser79) in cells treated with the indicated concentrations of TBM for $24 \mathrm{~h}$. $\beta$-actin was used as the internal control. i Cells were treated with TBM in the absence or presence of CC. AMPK, AMPK phosphorylation (Thr172) and LC3 were measured by immunoblot. j Cells were treated with TBM in the absence or presence CC for $24 \mathrm{~h}$. LC3 puncta formation was measured by immunofluorescence analysis. Scale bars: $20 \mu \mathrm{m}$. k Graph shows quantification of LC3-positive puncta in Figure J. ${ }^{*} p<0.05 ;{ }^{* *} p<0.01 ;{ }^{* *} p<0.001$
$\mathbf{E}$

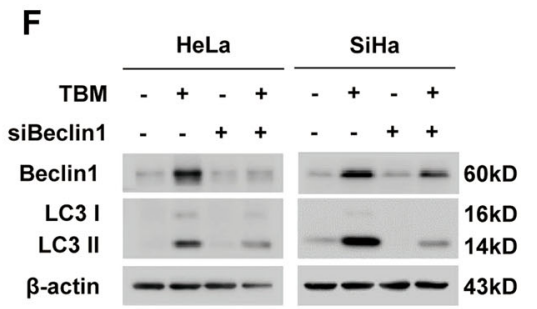

I

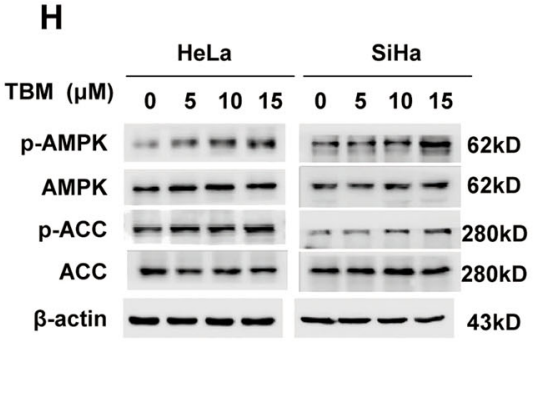

$\mathbf{K}$
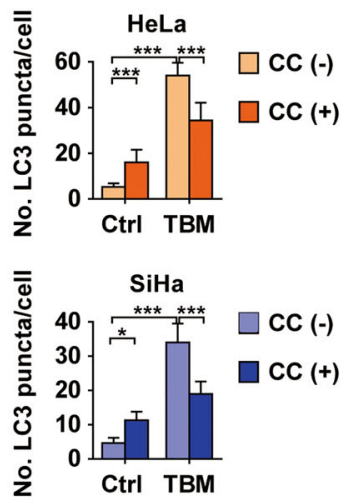
A

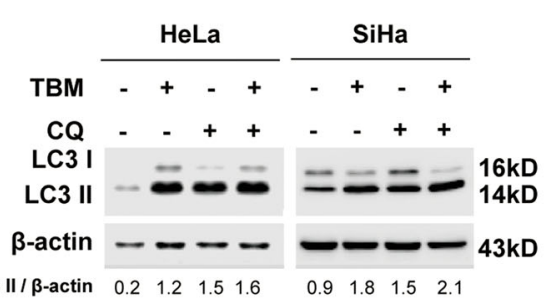

B

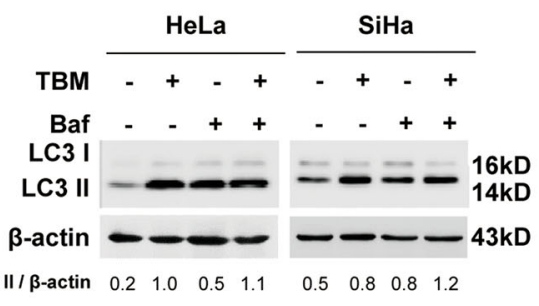

C

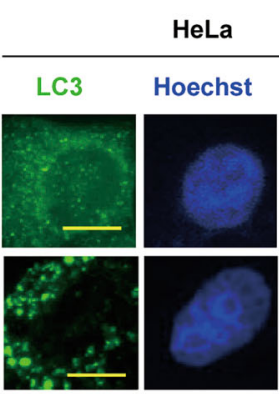

CQ

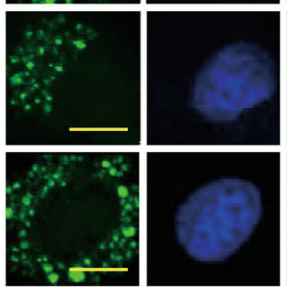

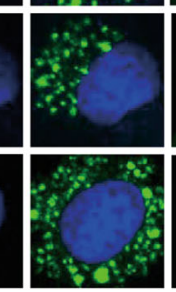

$\mathbf{F}$

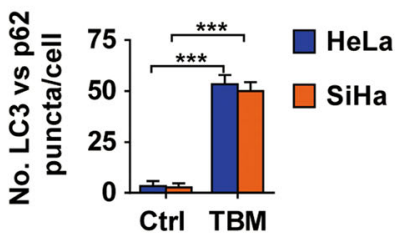

G

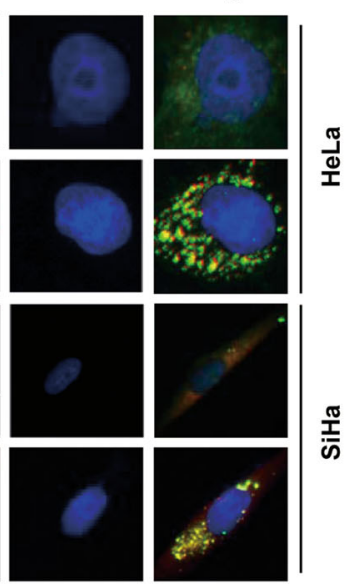

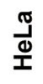

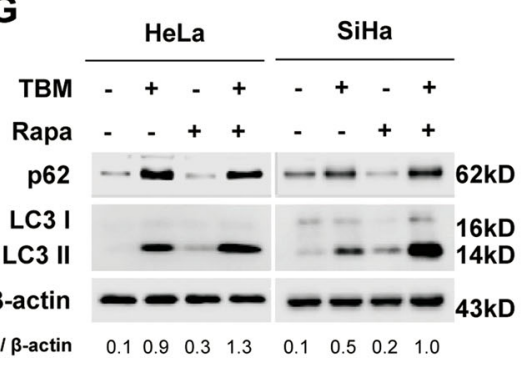

H

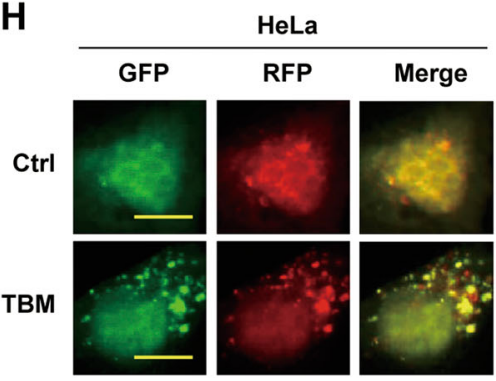

\begin{tabular}{lll}
\multicolumn{3}{c}{ SiHa } \\
\hline GFP & RFP & Merge
\end{tabular}
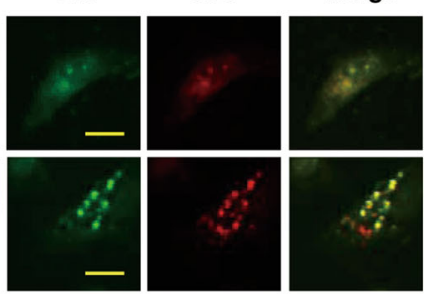

I

$\square$ Autophagolysosome $\overline{\bar{\delta}} \square$ Autophagosome

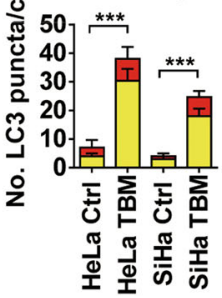

Fig. 5 (See legend on next page.) 
(see figure on previous page)

Fig. 5 TBM inhibits autophagic flux in cervical cancer cells. a HeLa and SiHa cells were treated with TBM in the absence or presence of CQ (10 $\mu M$ ). LC3 was measured with immunoblot. b Cells were treated with TBM in the absence or presence of bafilomycin A1 (Baf, $100 \mathrm{nM}$ ). LC3 was measured with immunoblot. c- $\mathbf{d}$ HeLa and SiHa cells were treated with TBM in the absence or presence of $10 \mu \mathrm{M}$ CQ. LC3 puncta formation was measured by immunofluorescence analysis and quantified by ImageJ. e-f Cells were treated with TBM. LC3 and p62 puncta were measured by immunofluorescence analysis and quantified by ImageJ. $\mathbf{g}$ HeLa and SiHa cells were treated with TBM in the absence or presence of rapamycin (500 nM). LC3 and p62 expression was measured with immunoblot. The ratio of LC3 I// $/$-actin was determined with ImageJ software. $\mathbf{h}-\mathbf{i}$ Cells were transfected with mCherry-GFP-LC3 for $48 \mathrm{~h}$, and treated with TBM for another $24 \mathrm{~h}$. The formation of autophagosome (mCherry-positive; GFPpositive) and autophagolysosome (mCherry-positive; GFP-negative) was examined and quantified by ImageJ. Scale bars: $20 \mu \mathrm{m} .{ }^{*} p<0.05 ;{ }^{* * *} p<$ 0.001

fluorescent autophagosomes and moderate increase of red fluorescent autophagolysosomes (Figs. 5h, i). In summary, these results show that autophagic flux inhibition plays a role in TBM-induced accumulation of autophagosomes.

\section{TBM blocks autophagic flux by impairing lysosomal enzyme}

The inhibition of autophagic flux could probably be attributed to impaired fusion between autophagosome and lysosome ${ }^{33}$. To determine whether lysosomal trafficking was affected by TBM, we assessed the colocalization of endogenous LC3 with LAMP1, which represents the formation of autophagolysosome ${ }^{34}$. Surprisingly, TBM induced a significant overlap between LC3 and LAMP1 and Pearson's correlation coefficient equaled about 0.5 (Supplementary Figure 7A and 7B), indicating that TBM induced fusion between autophagosome and lysosome. In line with the results, examination of the endogenous colocalization of LC3 and LysoTraker Red, a specific dye for lysosome labeling, also confirmed that autophagosome-lysosome fusion was not impaired by TBM (Supplementary Figure 7C and 7D).

Next, we detected whether TBM might deregulate the function of lysosome. We first examined the lysosomal $\mathrm{pH}$, as maintaining acidification is essential for lysosomal activity $^{35}$. However, AO staining displayed a significant increase of acidic vesicles in TBM-treated cells (Figs. 3g, h). To further monitor the lysosomal $\mathrm{pH}$, cells treated with TBM was applied with LysoSensor Green DND-189 (pKa 5.2) and analyzed with flow cytometry. As shown in Supplementary Figure 8A, an increasing shift of acidic compartments was observed in cells with TBM treatment, indicating the role of TBM in retaining the acidic environment. Similar result was obtained using LysoTracker Red (Supplementary Figure 8B), which accumulated in acidic lysosomes. These results indicate that TBMinduced autophagic inhibition is not correlated with lysosomal $\mathrm{pH}$ alteration.

Now that TBM maintained an adequate $\mathrm{pH}$ in cervical cancer cells, we next examined the expression of LAMP1, LAMP2, RAB5 and RAB7, which are critical membrane proteins for lysosome and endosome and are important for lysosomal physiology ${ }^{35}$. Results showed that all proteins exhibited an increase in TBM-treated cells on a dose-dependent manner (Fig. 6a), indicating that the disturbed autophagic flux was not due to the decreased membrane protein expression of lysosomes and endosomes.

In the end, we examined the function of lysosomal enzymes. Accumulating evidence indicated that dysfunction of lysosomal hydrolytic enzymes resulted in an increase of ubiquitinated proteins ${ }^{36}$. As expected, TBM treatment induced accumulation of ubiquitinated proteins in HeLa and SiHa cells (Fig. 6b). Furthermore, DQ-BSA was applied to monitor general endosomal-lysosomal process, during which the red fluorescence of DQ-BSA was supposed to self-quench without proteolytic cleavage $^{37}$. Notably, very little dequenching occurred in TBMtreated cells, indicating the TBM impaired lysosomal enzymes' activity (Fig. 6c). Similarly, the epidermal growth factor receptor (EGFR) degradation assay was performed in HeLa and SiHa cells. Accordingly, complex formed by EGF and its receptors might be endocytosed, and then transferred to lysosomes for degradation ${ }^{38}$. As shown in Fig. 6d, TBM inhibited EGF-triggered EGFR degradation, verifying the lysosomal dysfunction. In addition, we investigated the capacity of TBM on cathepsin processing in cervical cancer cells. Our data showed that TBM remarkably prevented the maturation of CTSD, resulting in inhibition of lysosomal activity (Fig. 6e). In summary, our data demonstrate that TBM inhibits lysosomal cathepsin activity, thereby leading to accumulation of impaired autophagolysosomes.

\section{Accumulation of impaired autophagolysosomes contributes to TBM-induced cell death}

Insufficient autophagy, which would cause accumulation of damaged proteins or organelles, may be a disaster to cancer cells and lead to cell death ${ }^{39}$. To investigate the link between autophagy blockade and cell death induced by TBM, we transfected cervical cancer cells with Beclin1 or ATG5 siRNA, followed by TBM treatment. Cell viability was measured with MTT assay and EdU labeling. These data demonstrated that reduction in ATG5 or Beclin1 levels rescued the cell viability caused by TBM (Figs. $7 \mathrm{a}-\mathrm{c}$ ). Furthermore, similar results were obtained 
A

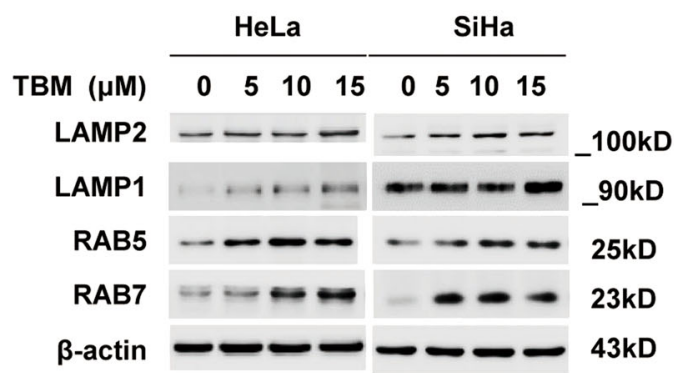

C

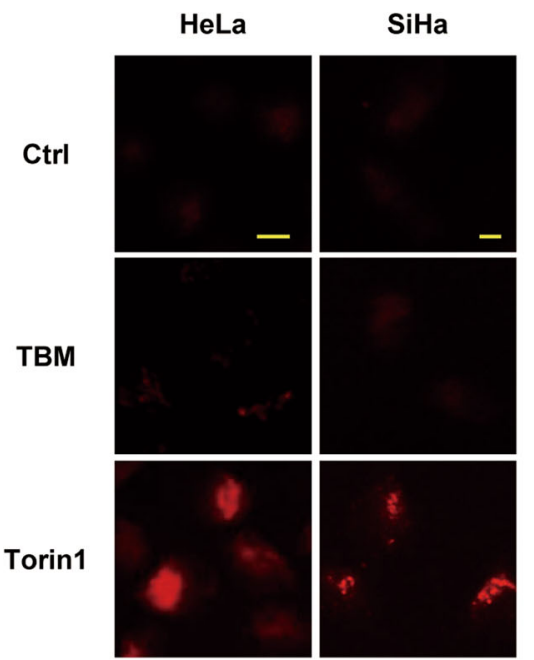

B

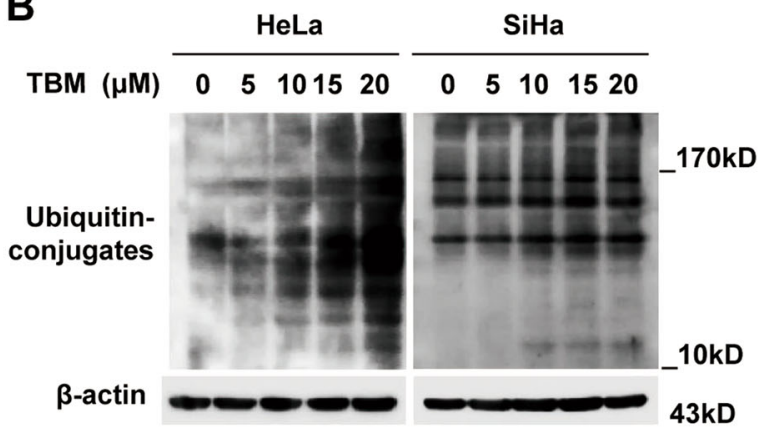

D

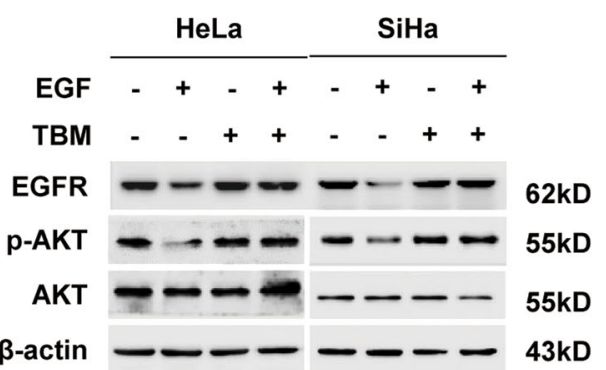

E

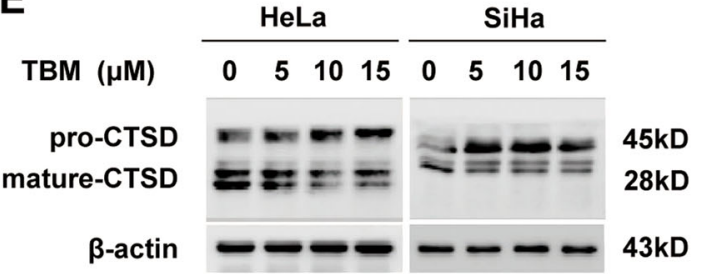

Fig. 6 TBM impairs lysosomal hydrolytic activity in cervical cancer cells. a Cells were treated with indicated concentrations of TBM for $24 \mathrm{~h}$. The expression of LAMP2, LAMP1, RAB5, and RAB7 in whole cell lysates was determined by immunoblot. b Immunoblot of ubiquitin in HeLa and SiHa cells treated with TBM in indicated concentrations for $24 \mathrm{~h}$. c Autophagolysosomes stained with DQ-BSA in HeLa and SiHa cells treated with $15 \mu \mathrm{M}$ TBM for $24 \mathrm{~h}$. Accumulation of fluorescent signal indicated the lysosomal proteolysis of DQ-BSA. Torin1 acted as positive control. Scale bar: $10 \mu \mathrm{M}$. $\mathbf{d}$ HeLa and SiHa cells were starved of serum overnight and treated with EGF (100 nM) in the absence or presence of $15 \mu \mathrm{M}$ TBM for $2 \mathrm{~h}$. EGFR, AKT and phosphorylation of AKT (Thr 308) were analyzed by immunoblot. e Immunoblot analysis of endogenous CTSD in HeLa and SiHa cells treated with 15 $\mu \mathrm{M}$ TBM for $24 \mathrm{~h}$

by using the autophagic inhibitors, 3-MA or WTM (Fig. 7d). Intriguingly, combination use of TBM with CQ or Baf A1 exacerbated TBM-induced cell death (Figs. 2a, b, 7e). In summary, these data indicate that TBM-induced autophagolysosome accumulation contributes to cell death in cervical cancer cells.

\section{TBM enhances chemotherapeutic sensitivity through autophagy modulation}

Previous studies have found that autophagy inhibition can enhance the efficacy of chemotherapeutic agents by abolishing the chemoresistance of cancer cells ${ }^{40,41}$. As a specific autophagy modulator, we therefore explored whether TBM would sensitize cervical cancer cells to several chemotherapeutic drugs, including CDDP, PTX, DOX and 5-FU. Co-treatment of HeLa and SiHa cells with TBM and CDDP or PTX in $24 \mathrm{~h}$, resulted in remarkable decrease in cell viability than that of DOX and 5-FU, whereas minimal cytotoxicity was observed when monotherapy was given (Fig. 8a). While co-treatment for 48 and $72 \mathrm{~h}$, TBM treatment combined with DOX or 5FU caused a more severe increase of cell death (Supplementary Figure 9). Furthermore, in one HPV negative human normal epithelial cell line (HaCat), TBM would significantly enhance the cytotoxicity in DOX-treated cells, but slightly in PTX, 5-FU and CDDP-treated cells, which is different with cancer cells (Supplementary Figure 10). These results suggest that low concentration of TBM, acting as a chemosensitizer or autophagy modulator, is relatively safe in combination treatment with some drugs, or may produce severe side reaction when co-treated with other drugs. 

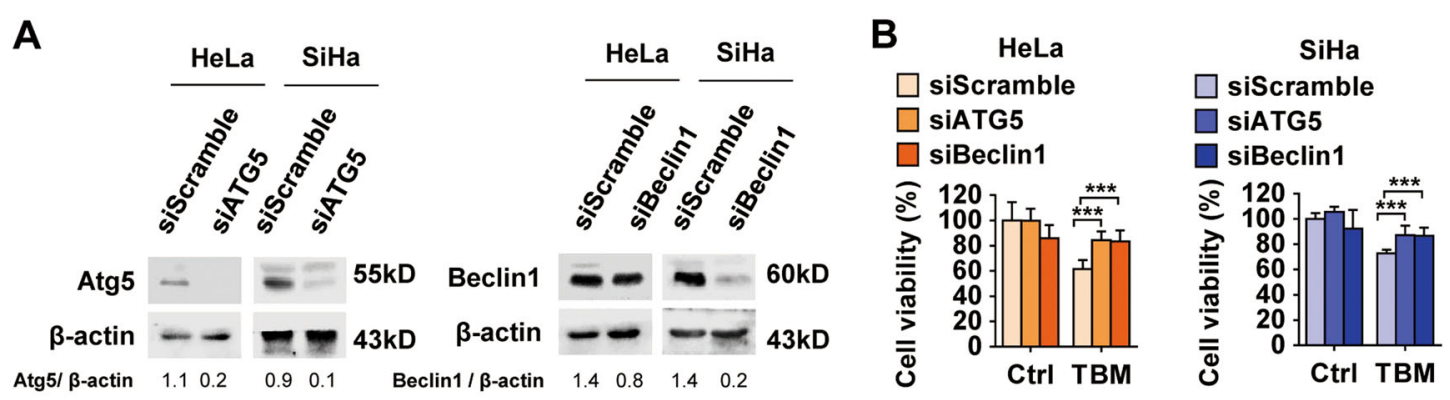

C

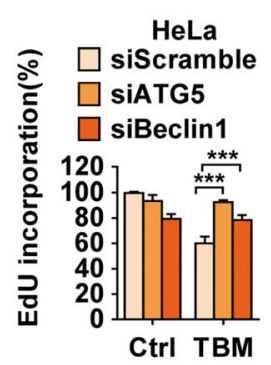

E

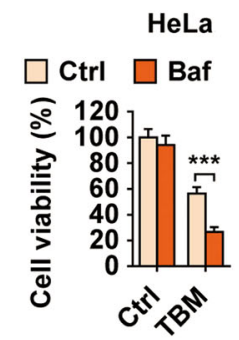

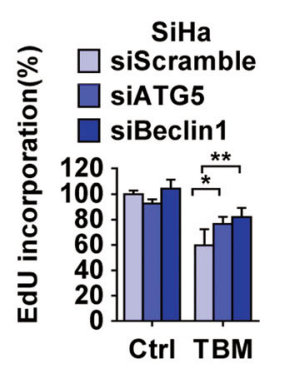

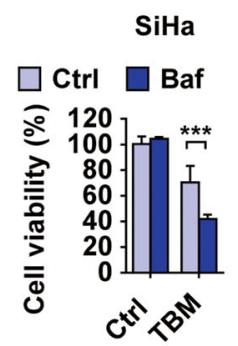

D

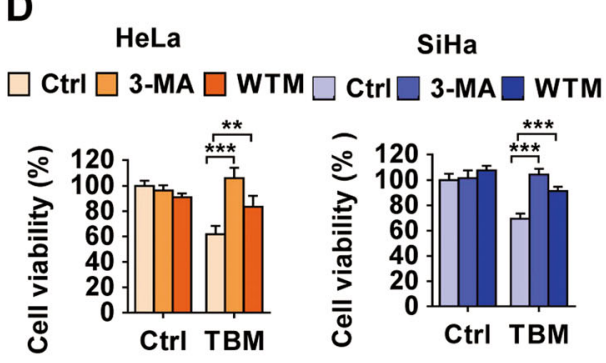

Fig. 7 Accumulation of impaired autophagolysosomes is contributed to TBM-induced cell death. a Cells were transfected with siScramble, siATG5 or siBeclin 1 for $48 \mathrm{~h}$. The expression of Atg 5 and Beclin 1 was measured by immunoblot. The ratio of Atg5/ $\beta$-actin and Beclin $1 / \beta$-actin was determined with ImageJ software. b-c Cells were transfected with siScramble, siATG5 or siBeclin 1 for $48 \mathrm{~h}$, and then treated with $15 \mu \mathrm{M}$ TBM for another $24 \mathrm{~h}$. b Cell viability was detected by MTT assay. c Proliferation rate was detected by EdU labeling. $\mathbf{d}$ Cells were treated with $15 \mu \mathrm{M}$ TBM in the absence or presence of 3-MA or WTM. Cell viability was determined by MTT assay. e Cells were treated with $15 \mu \mathrm{M}$ TBM in the absence or presence of bafilomycin A1 (Baf). Cell viability was determined by MTT assay. ${ }^{*} p<0.05 ;{ }^{* * *} p<0.001$

To reveal the underlying mechanism, we focused on the combination between TBM and CDDP, since CDDP is the first line drug in clinical cervical cancer treatment ${ }^{42}$. It has been reported that CDDP might induce a protective autophagy (Fig. 8b), a cause of chemoresistance ${ }^{42}$. As improvement of therapeutic efficacy may result from the defected autophagy induced by TBM, we aimed to examine whether autophagy blockade was involved in the combination strategies. As shown in Fig. 8c, increased LC3 II conversion by CDDP was further augmented in the presence of TBM. Meanwhile, CDDP-induced degradation of p62 was obstructed by TBM, suggesting that CDDP-induced autophagy flux was blocked by TBM and more impaired autophagolysosomes were accumulated. Furthermore, molecularly inhibiting autophagy by transfecting cervical cancer cells with ATG5 or Beclin1 siRNA decreased the LC3 II expression (Figs. 8d, e). Accordingly, in combined treatment, cell proliferation was partially restored by knockdown of ATG5 and Beclin1 (Fig. 8f), indicating that autophagy initiation was involved in TBMinduced accumulation of impaired autophagolysosomes and enhanced cytotoxicity. In summary, these data indicate that TBM may be a potent synergistic application as an autophagy modulator for cancer treatment.

\section{Discussion}

TBM, an ingredient from traditional Chinese herb, has been recently emerged to play a tumor-suppressive role in multiple cancers ${ }^{17,43-46}$. However, the molecular mechanisms have not been clearly determined. Here, we demonstrated that TBM was a potent autophagy modulator. TBM induced an AMPK-dependent autophagy initiation and inhibited autophagic degradation by inhibiting lysosomal proteolysis, leading to 

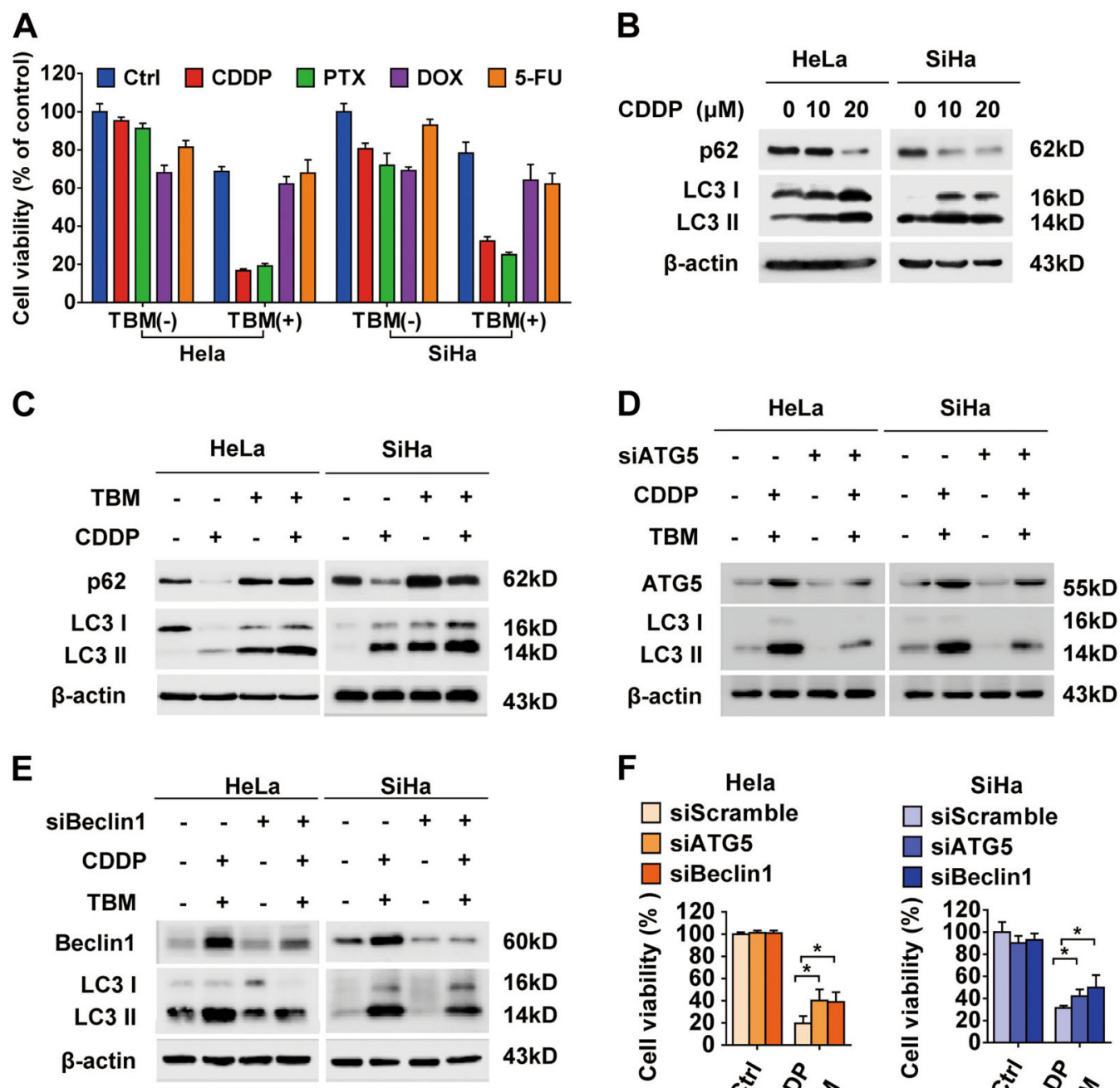

$60 \mathrm{kD}$
$16 \mathrm{kD}$
$14 \mathrm{kD}$
$43 \mathrm{kD}$
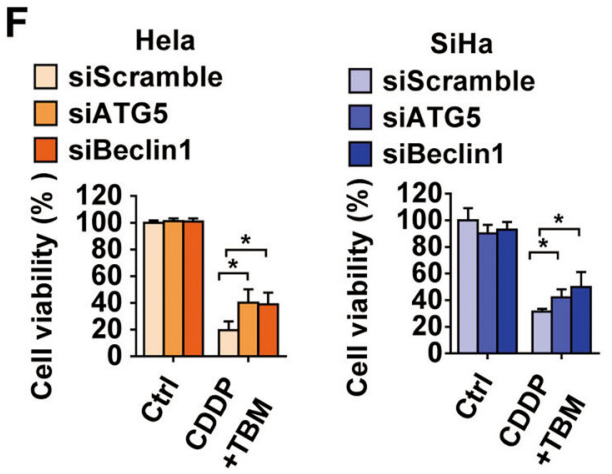

Fig. 8 TBM enhances chemotherapeutic sensitivity through autophagy modulation. a HeLa and SiHa cells were treated with chemotherapeutic drugs, including cisplatin (CDDP, $10 \mu \mathrm{M})$, paclitaxel (PTX, $100 \mathrm{nM})$, doxorubicin (DOX, $0.25 \mu \mathrm{M})$ and 5-fluorouracil $(5-\mathrm{FU}, 2 \mu \mathrm{M})$ in the absence or presence of $10 \mu \mathrm{M}$ TBM for $24 \mathrm{~h}$ and MTT assays were performed to assess cell viability. $\mathbf{b}$ Cells were treated with CDDP in indicated concentrations for $24 \mathrm{~h}$, and the expression of LC3 and p62 was measured with immunoblot. $\mathbf{c}$ HeLa and SiHa cells were treated with $10 \mu \mathrm{M}$ CDDP in the absence or presence of TBM for $24 \mathrm{~h}$, and the expression of LC3 and p62 was measured with immunoblot. $\mathbf{d}$-e Cells transfected with siScramble, siATG5 or siBeclin 1 were co-treated without or with TBM and CDDP, and then subjected to monitor the expression of LC3 II conversion. $\mathbf{f}$ Cells transfected with siScramble, siATG5, or siBeclin1 were co-treated without or with TBM and CDDP. Cell viability was determined by MTT assay. ${ }^{*} p<0.05$

autophagolysosomes accumulation in cervical cancer cells. Meanwhile, our findings indicated that accumulation of immature autophagolysosomes by TBM triggered cell death, and enhanced CDDP-mediated cytotoxicity in cervical cancer cells.

Autophagy is a basic catabolic process, which captures unnecessary or dysfunctional cellular components and fuses with lysosomes for degradation ${ }^{34}$. The function of autophagy in cancer therapy is controversial ${ }^{10,11}$. For one hand, autophagy may contribute to cell death; ${ }^{47,48}$ for the other hand, autophagy may play a supportive role in drug resistance ${ }^{49}$. In this study, we demonstrated that TBM could induce biosynthesis of autophagosome, which was subsequently engulfed by the lysosomes to form autophagolysosomes. However, the autophagolysosomes failed to degrade the cargo due to defects of lysosomal function. Inhibition of autophagy initiation restored cell proliferation, while autophagolysosome inhibitors aggravated the cell death incidence, suggesting that TBM functions as a promising antitumor agent by inducing a particular autophagy inhibiting effect. 
The AMPK signaling pathway is a well-recognized pathway accounting for autophagy ${ }^{50}$. As one of the major metabolic sensors, AMPK plays an important role in modulation of the autophagy process. Traditionally, AMPK triggers autophagy by either directly activating unc-51 like autophagy activating kinase 1 (ULK1) or indirectly inhibiting mechanistic target of rapamycin (mTOR), both of which eventually lead to activation of Beclin1-Vps34 complex through phosphorylating Beclin $1^{51}$. We here demonstrated that TBM remarkably decreased ATP production, thereby inducing phosphorylation of AMPK and activation of Beclin1-Vps34 complex to initiate autophagy. In addition to initiation of autophagy, increasing studies have revealed the role of AMPK in the late stage of autophagy, such as autophagic proteolysis. For one hand, AMPK can increase the cellular ATP levels to promote autophagic degradation ${ }^{52}$. For the other hand, autophagy flux may also be inhibited with AMPK activation when intracellular nutrition depletion is too excessive, as the autophagy requires adequate amount of ATP to complete this complicated membrane flowdependent process $^{53}$. Thus, the mechanism of AMPKmediated autophagy under TBM treatment is complex and remains to be further elucidated.

Lysosome has been considered as the degradation center in most eukaryotic cells ${ }^{54}$. Maintaining lysosomal physiology is essential for autophagy. For instance, the lysosomal acidification, lysosomal trafficking, lysosome fusion with late endosome or autophagosome, and maturation of lysosomal proteases are the most important impacts for lysosomal physiology ${ }^{55}$. In our study, the disruption of lysosomal activity induced by TBM was due to the dysfunction of lysosomal enzyme. In addition, a stale lysosomal function is essential for complete autophagy and cellular homeostasis, especially in tumor cells suffering from stress condition, in contrast, accumulation of autophagolysosomes due to defect lysosomal activity will result cancer cells death ${ }^{56,57}$. It is therefore conceivable that the late stage inhibition of autophagy is contributed to the cytotoxicity of TBM in cervical cancer cells. However, further investigation is needed to be performed on the detailed mechanism of impaired lysosomal proteolysis by TBM.

Recently, the importance of interaction between drug resistance and autophagy in cancer treatment has been widely emerged. Currently, autophagy inhibitors can be classified into early-stage inhibitors (wortmannin, LY294002 and 3-MA) and late-stage inhibitors (CQ, HCQ and Baf $\mathrm{A} 1)^{41}$. Extensive preclinical studies have demonstrated that autophagy inhibition could enhance the treatment efficacy of chemotherapeutic drugs towards cancer $^{26,40}$, so novel autophagy inhibitors are worthwhile to be exploited. In this study, we found that TBM markedly promoted the sensitivity of CDDP in cervical cancer cells via modulating autophagy. Remarkably, considering that TBM displayed certain antitumor bioactivity, combination of TBM with other chemotherapeutic drugs might be more effective in cancer therapy.

In summary, our study demonstrates that TBM triggers cell death and promotes CDDP sensitivity in cervical cancer by inducing the accumulation of impaired autophagolysosomes. Our findings suggest that TBM is a specific autophagy modulator and could be potentially developed as an adjuvant for further cancer treatment. Our research provides a basis for potential use of TBM in further cancer therapy, especially in cervical cancer treatment.

\section{Materials and methods \\ Cell lines and culture}

Human cervical cancer cell lines HeLa and SiHa, glioblastoma cell lines U87 and U251, colorectal cancer cell lines HCT116, HT29 and SW480, hepatocarcinoma cell lines HepG2 and Hep3B, lung cancer cell lines A549 and H1299, human intestinal epithelial cell line HIEC, as well as human lung fibroblasts cells MRC5 were obtained from the American Type Culture Collection (ATCC). Huh7 cell was purchased from the Type Culture Collection of the Chinese Academy of Sciences. Human breast cancer cell lines MCF-7 and MDA-MB-468 were provided by Professor Qiang Yu (Genome Institute of Singapore). Human normal epithelial cell line HaCat cells was provided by Professor Qunying Lei (Fudan University of China). Human fetal normal liver cells LO2 cells were obtained from our lab storage. All cells were cultured in Dulbecco's modified Eagle's medium or RPMI1640 (Invitrogen, Carlsbad, CA, USA), supplemented with $10 \%$ fetal bovine serum (FBS; Gibco, 10100-147), $10^{5} \mathrm{U} / \mathrm{L}$ penicillin, and $100 \mathrm{mg} / \mathrm{L}$ streptomycin at $37^{\circ} \mathrm{C}$ in an atmosphere containing $5 \% \mathrm{CO}_{2}$.

\section{Cell viability measurement}

Cells were treated with TBM alone or combination with other drugs. After treatment, $0.5 \mathrm{mg} / \mathrm{mL} 3$-(4, 5-dimethyl2-thiazolyl)-2, 5-diphenyl-2-H-tetrazolium bromide (MTT) was added and incubated for $4 \mathrm{~h}$. The insoluble formazan product of cells was dissolved in dimethyl sulfoxide after three times washing with phosphate buffered saline (PBS). The optical density (OD) of each culture well was measured by spectrophotometry at $570 \mathrm{~nm}$. The OD value of the control cells was taken as $100 \%$ viability. Cellular membrane integrity was assessed with the lactic dehydrogenase (LDH) Release kit (Beyotime), as described previously ${ }^{58}$. Briefly, LDH activity was assayed by adding $100 \mu \mathrm{L}$ potassium phosphate buffer within 23 $\mathrm{mM}$ pyruvate and $0.3 \mathrm{mg} / \mathrm{mL} \beta-\mathrm{NADH}$. Then the conversion of NADH to $\mathrm{NAD}^{+}$was monitored at $340 \mathrm{~nm}$ with a spectrophotometer. 


\section{Flow cytometry}

Cell apoptosis was analyzed using a PI/Annexin V kit (KeyGEN Biotech). Cells were washed once with PBS, and resuspended in PI//Annexin V solution. At least 20000 live cells were analyzed. The flow cytometry data were collected with FACSCalibur flow cytometer (Becton Dickinson, San Jose, CA, USA), and then analyzed with FlowJo software.

\section{Tumor xenograft model}

The study was subjected to the Institutional Animal Care and Treatment Committee of Sichuan University. Female BALB/c nude mice at 4 weeks of age were obtained from Beijing HFK Bioscience and kept in a sterile environment. HeLa cells $\left(5 \times 10^{6}\right)$ resuspended in $100 \mu \mathrm{L}$ PBS were injected subcutaneously into the right dorsal region of each mouse, respectively. Seven days after implantation, mice were randomly divided into 2 groups ( $n=5$ /group). (1) Control, receiving daily i.p. saline solution (NS); (2) TBM, receiving daily i.p. $3 \mathrm{mg} / \mathrm{kg}$ of TBM. Tumor volumes were measured every 2 days determined by measuring the length (l) and the width (w) and calculating the volume $\left(\mathrm{V}=1 \times \mathrm{w}^{2} / 2\right)$. After 16 days of treatment, mice were sacrificed. Tumor tissues were isolated and either frozen in liquid nitrogen or fixed in $10 \%$ formalin immediately.

\section{Immunohistochemical analysis}

Formalin fixed tissues were embedded by paraffin, and sections were consecutively cut $(4 \mu \mathrm{m}$ thickness). The paraffin-sections were dewaxed, rehydrated, and incubated in $3 \% \mathrm{H}_{2} \mathrm{O}_{2}$ for 10 min to quench the endogenous peroxidase activity. After antigen retrieval in citrate buffer and incubating with normal rabbit serum for $20 \mathrm{~min}$ at 37 ${ }^{\circ} \mathrm{C}$, the tumor sections were performed with indicated antibodies, following by reaction with diaminobenzidine (Fuzhou Maixin Biotechnology, DAB-0031) and counterstaining with Mayer hematoxylin (Beyotime, C0107). Imaging was obtained with a Leica DM2500 microscope. Immunohistochemical staining was assessed by both the fraction of positive cells $(0-100 \%)$ and the immunostaining intensity (0-negative, 1-weak, 2-moderate, 3strong). The final score was calculated by multiplying the fraction score and the intensity score.

\section{Immunoblot and Immunoprecipitation}

Cells were solubilized in lysis buffer and lysates were centrifuged. Supernatant fractions were separated by SDS-PAGE and transferred to polyvinylidene difluoride membranes (EMD Millipore, ISEQ00010). Immunoreactive bands were detected by ECL (EMD Millipore, WBKLS0500). For immunoprecipitations, cell lysates were incubated with $1 \mu \mathrm{g}$ indicated antibodies overnight at $4{ }^{\circ} \mathrm{C}$, following by addition of protein A-Sepharose beads ( $40 \mu \mathrm{L}, \mathrm{GE}$ Healthcare) for another $2 \mathrm{~h}$. The samples were analyzed by immunoblot.

\section{Lysosomal pH measurement}

Cells were loaded with $1 \mathrm{mM}$ LysoSensor Green DND189 (Invitrogen, 10010-023) or LysoTracker Red (C1046) in pre-warm regular medium for $20 \mathrm{~min}$ at $37^{\circ} \mathrm{C}$. Then the cells were washed twice with PBS and immediately analyzed by flow cytometry. The flow cytometry data were collected with FACSCalibur flow cytometer (Becton Dickinson, San Jose, CA, USA), and then analyzed with FlowJo software.

\section{DQ-BSA degradation assay}

Cells were plated onto glass coverslips, and incubated with $10 \mathrm{mg} / \mathrm{mL}$ DQ-BSA (Thermo Fisher Scientific, D12051) for 30 minutes. After washing with PBS for three times, the fluorescent signal of DQ-BSA was recorded with a Leica DM2500 microscope.

\section{Statistical analysis}

All data are reported as mean \pm SD. Data analysis was performed using Prism 5.0 (Graph-Pad Software, Inc., La Jolla, CA). The statistical significance of the difference between experimental groups in instances of single comparisons was determined using the 2-tailed unpaired Student $\mathrm{t}$ test of the means. Comparisons where $p<0.05$ were deemed significant.

\section{Acknowledgements}

The authors thank Prof. Qiang Yu and Prof. Qunying Lei for providing cell lines used in this study. This work was supported by grants from the Chinese NSFC $(81872014,81430071,81672381$ and 81602194), the National Key Research and development program of China (2016YFC1200203), Chongqing Natural Science Foundation (cstc2016jcyjA0227), and the Science and Technology Research Program of Chongqing Municipal Education Commission (Grant No. KJQN201800429).

\section{Author details}

'Department of Biochemistry and Molecular Biology, and Molecular Medicine and Cancer Research Center, Chongqing Medical University, Chongqing 400016, P.R. China. ${ }^{2}$ State Key Laboratory of Biotherapy and Cancer Center, West China Hospital, and West China School of Basic Medical Sciences \& Forensic Medicine, Sichuan University, and Collaborative Innovation Center for Biotherapy, Chengdu 610041, P.R. China. ${ }^{3}$ School of Biological Sciences and Technology, Chengdu Medical College, Chengdu 610083, P.R. China. ${ }^{4}$ School of pharmacy, Xinxiang Medical University, Xinxiang 453003, P. R. China. ${ }^{5}$ Department of Oncology, The First Affiliated Hospital of Chongqing Medical University, Chongqing 400016, P. R. China. ${ }^{6}$ Department of Neurology, The Affiliated Hospital of Hainan Medical College, Hainan 570102, P.R. China. 'West China School of Basic Medical Sciences \& Forensic Medicine, Sichuan University, Chengdu 610041, P.R. China

\section{Conflict of interest}

The authors declare that they have no conflict of interest.

\section{Publisher's note}

Springer Nature remains neutral with regard to jurisdictional claims in published maps and institutional affiliations. 
Supplementary Information accompanies this paper at (https://doi.org/ 10.1038/s41419-018-1151-3).

Received: 24 May 2018 Revised: 28 September 2018 Accepted: 9 October 2018

Published online: 02 November 2018

\section{References}

1. Torre, L. A. et al. Global cancer statistics, 2012. CA Cancer J. Clin. 65, 87-108 (2015).

2. Ginsburg, O. et al. The global burden of women's cancers: a grand challenge in global health. Lancet 389, 847-860 (2017).

3. Islam, R. M., Billah, B., Hossain, M. N. \& Oldroyd, J. Barriers to cervical cancer and breast cancer screening uptake in low-income and middle-income countries: a systematic review. Asian Pac. J. Cancer Prev. 18, 1751-1763 (2017).

4. Denny, L. et al. Interventions to close the divide for women with breast and cervical cancer between low-income and middle-income countries and highincome countries. Lancet 389, 861-870 (2017).

5. Takebayashi, K. et al. Comparison of curative surgery and definitive chemoradiotherapy as initial treatment for patients with cervical esophageal cancer. Dis. Esophagus 30, 1-5 (2017).

6. Zhu, L. et al. Predicting and early monitoring treatment efficiency of cervical cancer under concurrent chemoradiotherapy by intravoxel incoherent motion magnetic resonance imaging. J. Comput. Assist. Tomogr. 41, $422-429$ (2017).

7. Morel, E. et al. Autophagy: a druggable process. Annu. Rev. Pharmacol. Toxicol. 57, 375-398 (2017)

8. Mizushima, N. A brief history of autophagy from cell biology to physiology and disease. Nat. Cell Biol. 20, 521-527 (2018).

9. Kimmelman, A. C. \& White, E. Autophagy and tumor metabolism. Cell Metab. 25, 1037-1043 (2017).

10. White, E. Deconvoluting the context-dependent role for autophagy in cancer Nat. Rev. Cancer 12, 401-410 (2012).

11. Moosavi, M. A. et al. Phytochemicals as potent modulators of autophagy for cancer therapy. Cancer Lett. 424, 46-69 (2018).

12. Lei, $Y$. et al. Targeting autophagy in cancer stem cells as an anticancer therapy. Cancer Lett. 393, 33-39 (2017).

13. Kasai, R. et al. Tubeimoside I, a new cyclic bisdesmoside from Chinese cucurbitaceous folk medicine "tu bei mu", a tuber of Bolbostemma paniculatum. Chem. Pharm. Bull. 34(9), 3974-3977 (1986).

14. Zhang, Y., Xu, X. \& He, P. Tubeimoside-1 inhibits proliferation and induces apoptosis by increasing the $\mathrm{Bax}$ to $\mathrm{BCl}-2$ ratio and decreasing $\mathrm{COX}-2$ expression in lung cancer A549 cells. Mol. Med. Rep. 4, 25-29 (2011).

15. Zhang, Y. et al. Effects of tubeimoside-1 on the proliferation and apoptosis of BGC823 gastric cancer cells in vitro. Oncol. Lett. 5, 801-804 (2013).

16. Jia, G. et al. Tubeimoside-1 induces glioma apoptosis through regulation of Bax/BCl-2 and the ROS/Cytochrome C/Caspase-3 pathway. Onco. Targets Ther. 8, 303-311 (2015).

17. Yang, J. B. et al. Tubeimoside-1 induces oxidative stress-mediated apoptosis and G0/G1 phase arrest in human prostate carcinoma cells in vitro. Acta Pharmacol. Sin. 37, 950-962 (2016).

18. Liu, H. Z. et al. Tubeimoside I sensitizes cisplatin in cisplatin-resistant human ovarian cancer cells (A2780/DDP) through down-regulation of ERK and upregulation of p38 signaling pathways. Mol. Med. Rep. 4, 985-992 (2011).

19. Muzaffar, S. \& Chattoo, B. B. Apoptosis-inducing factor (Aif1) mediates anacardic acid-induced apoptosis in Saccharomyces cerevisiae. Apoptosis 22, 463-474 (2017).

20. Xie, Y. et al. Ferroptosis: process and function. Cell Death Differ. 23, 369-379 (2016).

21. Sun, W. et al. Cytosolic calcium mediates RIP1/RIP3 complex-dependent necroptosis through JNK activation and mitochondrial ROS production in human colon cancer cells. Free Radic. Biol. Med. 108, 433-444 (2017).

22. Cao, K. \& Tait, S. W. G. Apoptosis and cancer: force awakens, phantom menace, or both?. Int Rev Cell Mol Biol 337, 135-152 (2018).

23. Lee, Y. K. et al. Development of LC3/GABARAP sensors containing a LIR and a hydrophobic domain to monitor autophagy. EMBO J. 36, 1100-1116 (2017).

24. Klionsky, D. J. et al. Guidelines for the use and interpretation of assays for monitoring autophagy (3rd edition. Autophagy 12, 1-222 (2016).
25. Munson, M. J. \& Ganley, I. G. MTOR, PIK3C3, and autophagy: Signaling the beginning from the end. Autophagy 11, 2375-2376 (2015).

26. Dikic l., Elazar Z. Mechanism and medical implications of mammalian autophagy. Nat. Rev. Mol. Cell Biol. 19, 349-364 (2018).

27. Wang, Y. et al. Natural plant extract tubeimoside I induces cytotoxicity via the mitochondrial pathway in human normal liver cells. Mol. Med. Rep. 4, 713-718 (2011).

28. Wang, F., Ma, R. \& Yu, L. Role of mitochondria and mitochondrial cytochrome $\mathrm{c}$ in tubeimoside I-mediated apoptosis of human cervical carcinoma HeLa cell line. Cancer Chemother. Pharmacol. 57, 389-399 (2006).

29. Shen, Q. W., Gerrard, D. E. \& Du, M. Compound C, an inhibitor of AMPactivated protein kinase, inhibits glycolysis in mouse longissimus dorsi postmortem. Meat Sci. 78, 323-330 (2008).

30. Wang, C. et al. Elevated p62/SQSTM1 determines the fate of autophagydeficient neural stem cells by increasing superoxide. J. Cell Biol. 212, 545-560 (2016).

31. Mauvezin, C. \& Neufeld, T. P. Bafilomycin A1 disrupts autophagic flux by inhibiting both V-ATPase-dependent acidification and Ca-P60ASERCAdependent autophagosome-lysosome fusion. Autophagy 11, 1437-1438 (2015).

32. $\mathrm{Ye}, \mathrm{H}$. et al. Chloroquine, an autophagy inhibitor, potentiates the radiosensitivity of glioma initiating cells by inhibiting autophagy and activating apoptosis. BMC. Neurol. 16, 178 (2016).

33. Shen, H. M. \& Mizushima, N. At the end of the autophagic road: an emerging understanding of lysosomal functions in autophagy. Trends Biochem. Sci. 39, 61-71 (2014).

34. Devkota, S. The autophagy process. Oncotarget 8, 18623 (2017).

35. Eskelinen, E.-L., Tanaka, Y. \& Saftig, P. At the acidic edge: emerging functions for lysosomal membrane proteins. Trends Cell Biol. 13, 137-145 (2003).

36. van der Veen, A. G. \& Ploegh, H. L. Ubiquitin-like proteins. Annu. Rev. Biochem. 81, 323-357 (2012)

37. Frost, L. S., Dhingra, A., Reyes-Reveles, J. \& Boesze-Battaglia, K. The use of DQBSA to monitor the turnover of autophagy-associated cargo. Methods Enzymol. 587, 43-54 (2017).

38. Shinde, S. R. \& Maddika, S. PTEN modulates EGFR late endocytic trafficking and degradation by dephosphorylating Rab7. Nat. Commun. 7, 10689 (2016).

39. Zhang, L. et al. Redox signaling: potential arbitrator of autophagy and apoptosis in therapeutic response. Free Radic. Biol. Med. 89, 452-465 (2015).

40. Rubinsztein, D. C., Codogno, P. \& Levine, B. Autophagy modulation as a potential therapeutic target for diverse diseases. Nat. Rev. Drug. Discov. 11, 709-730 (2012).

41. Rubinsztein, D. C., Gestwicki, J. E., Murphy, L. O. \& Klionsky, D. J. Potential therapeutic applications of autophagy. Nat. Rev. Drug. Discov. 6, 304-312 (2007).

42. Boussios, S. et al. Management of patients with recurrent/advanced cervical cancer beyond first line platinum regimens: where do we stand? A literature review. Crit. Rev. Oncol. Hematol. 108, 164-174 (2016).

43. Peng, Y., Zhong, Y. \& Li, G. Tubeimoside-1 suppresses breast cancer metastasis through downregulation of CXCR4 chemokine receptor expression. BMB. Rep. 49, 502-507 (2016).

44. Bian, Q., Liu, P., Gu, J. \& Song, B. Tubeimoside-1 inhibits the growth and invasion of colorectal cancer cells through the Wnt/beta-catenin signaling pathway. Int. J. Clin. Exp. Pathol. 8, 12517-12524 (2015).

45. Hao, W., Wang, S. \& Zhou, Z. Tubeimoside-1 (TBMS1) inhibits lung cancer cell growth and induces cells apoptosis through activation of MAPK-JNK pathway. Int. J. Clin. Exp. Pathol. 8, 12075-12083 (2015).

46. Xu, Y., Chiu, J. F., He, Q. Y. \& Chen, F. Tubeimoside-1 exerts cytotoxicity in HeLa cells through mitochondrial dysfunction and endoplasmic reticulum stress pathways. J. Proteome Res. 8, 1585-1593 (2009).

47. Liu, R. et al. Itraconazole suppresses the growth of glioblastoma through induction of autophagy: involvement of abnormal cholesterol trafficking. Autophagy 10, 1241-1255 (2014).

48. Dou, Q. et al. Ivermectin induces cytostatic autophagy by blocking the PAK1/ Akt axis in breast cancer. Cancer Res. 76, 4457-4469 (2016).

49. Wang, K et al. Quercetin induces protective autophagy in gastric cancer cells: involvement of Akt-mTOR- and hypoxia-induced factor 1alpha-mediated signaling. Autophagy 7, 966-978 (2011).

50. Wang, Z., Wang, N., Liu, P. \& Xie, X. AMPK and cancer. EXS 107, 203-226 (2016).

51. Kim, J., Kundu, M., Viollet, B. \& Guan, K. L. AMPK and mTOR regulate autophagy through direct phosphorylation of Ulk1. Nat. Cell Biol. 13, 132-141 (2011). 
52. Xie, N. et al. PRKAAAMPK restricts HBV replication through promotion of autophagic degradation. Autophagy 12, 1507-1520 (2016).

53. Meley, D. et al. AMP-activated protein kinase and the regulation of autophagic proteolysis. J. Biol. Chem. 281, 34870-34879 (2006).

54. Luzio, J. P., Pryor, P. R. \& Bright, N. A. Lysosomes: fusion and function. Nat. Rev. Mol. Cell Biol. 8, 622-632 (2007).

55. Hamalisto, S. \& Jaattela, M. Lysosomes in cancer-living on the edge (of the cell). Curr. Opin. Cell Biol. 39, 69-76 (2016).
56. Dielschneider, R. F., Henson, E. S. \& Gibson, S. B. Lysosomes as oxidative targets for cancer therapy. Oxid. Med. Cell Longev. 2017, 3749157 (2017)

57. Towers, C. G. \& Thorburn, A. Targeting the lysosome for cancer therapy. Cancer Discov. 7, 1218-1220 (2017).

58. Kim, Y. et al. BIX-01294 induces autophagy-associated cell death via EHMT2/ G9a dysfunction and intracellular reactive oxygen species production. Autophagy 9, 2126-2139 (2013). 